\title{
Téoros
}

Revue de recherche en tourisme

\section{Les Canadiens dans la Caraïbe}

Le triangle du tourisme de masse

\section{Bruno Sarrasin}

Volume 26, numéro 1, printemps 2007

Tourisme dans la Caraïbe : logiques régionales et perspectives de développement

URI : https://id.erudit.org/iderudit/1070992ar

DOI : https://doi.org/10.7202/1070992ar

Aller au sommaire du numéro

Éditeur(s)

Université du Québec à Montréal

ISSN

0712-8657 (imprimé)

1923-2705 (numérique)

Découvrir la revue

Citer cet article

Sarrasin, B. (2007). Les Canadiens dans la Caraïbe : le triangle du tourisme de masse. Téoros, 26(1), 29-32. https://doi.org/10.7202/1070992ar d'utilisation que vous pouvez consulter en ligne.

https://apropos.erudit.org/fr/usagers/politique-dutilisation/ 


\section{Les Canadiens dans la Caraïbe Le triangle du tourisme de masse}

\section{Bruno Sarrasin}

Les destinations touristiques de la Carailbe sont aux Canadiens ce que le Maghreb touristique est aux Européens : le soleil, la plage et la détente à bas prix dans un espace géographique accessible - quatre heures de vol et moins à partir de Toronto, Montréal ou Calgary -, où les destinations standardisées en formule «tout compris » sont interchangeables en fonction des modes et des saisons. Mais ce "paradis des vacances" cache une relation visiteurs / visités ténue sinon inexistante et des retombées économiques mitigées... pourtant, certaines îles de cette région voient l'affluence canadienne augmenter de façon importante depuis les cinq dernières années. La Caraïbe serait-elle devenue la nouvelle Floride des Canadiens et des Québécois? Après avoir identifié les principales destinations visitées par cette clientèle, le présent article propose de mieux comprendre qui sont les Canadiens qui voyagent dans cette région en présentant leurs principales caractéristiques et motivations de voyages. L'observation de séries statistiques longues permettra d'identifier quelques tendances lourdes dans le comportement de vacances et d'esquisser quelques hypothèses sur leur évolution. Enfin, nous proposons d'explorer quelques effets de la clientèle canadienne sur les environnements visités.

\section{Trois pays... une destination !}

Le graphique 1 montre l'évolution indicielle des arrivées de touristes en provenance du Canada vers l'ensemble de la Caraïbe, toutes destinations confondues. Suivant une évolution presque linéaire avec un taux d'accroissement annuel moyen (TAAM) ${ }^{1}$ de $5,29 \%$ au cours des 25 dernières années, la croissance des arrivées s'est accélérée en
2004 avec 36,6 \% de plus d'arrivées qu'en 2002. Cela correspond globalement aux cycles de l'économie canadienne avec une baisse annuelle moyenne de 6,68 \% pour la période 1980-1982; une stagnation en 1988-1989 (+0,58\%) et en 1995-1997 (TAAM de +0,16\%), avec une relance à partir de 1998 que les attentats du 11 septembre ont à peine freinée. L'année 2003, qui représente $200 \%$ d'augmentation du marché canadien depuis 1980, est d'ailleurs qualifiée par les principaux acteurs de l'industrie québécoise de "retour à la forme" du segment voyage-vacances (Bérubé, 2003). Les Transat, Vacances Signature et autres Skyservice ont maintenu un « ratio offre-demande acceptable », c'est-à-dire que la croissance de l'offre a été moins rapide que celle de la demande, de sorte que les ventes et les profits ont été en hausse notable pour l'ensemble des acteurs de l'industrie. L'offre de forfaits « tout compris » à destination de la Caraïbe est contrôlée par quelques joueurs seulement dans l'univers québécois et canadien du voyage et le marché des destinations soleil montre, à quelques exceptions près, une étonnante stabilité. Sam Char, responsable du développement des activités commerciales au Québec du voyagiste Signature, résumait de la façon suivante la situation qui prévalait en 2003 :

Les francophones ne vont pas dans les Antilles anglaises, ou peu. Quant à la Guadeloupe, c'est trop cher. Au Québec, sauf pour les périodes de pointe, il y a un prix plafond, de 1200 \$à 1500 \$ par semaine, qu'on ne veut pas dépasser. Quant au temps de vol préféré, il dépasse rarement les quatre heures. Nous ajoutons le Costa Rica cette année, avec sa durée de vol de cinq heures et demie. Il faudra voir quelle sera la réaction. (Bérubé, 2003)

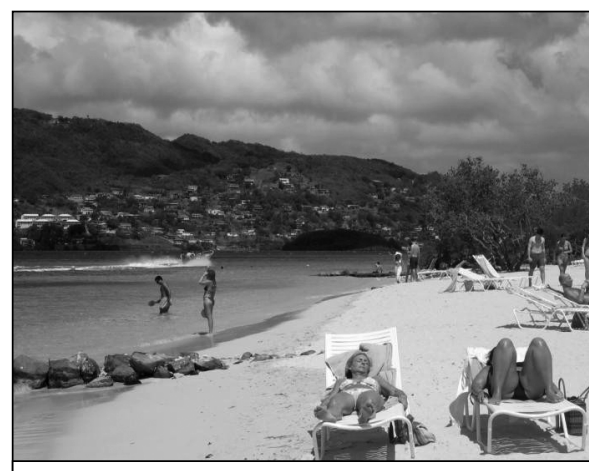

Sainte-Lucie, un monde sans hiver. Des plages appréciées par les retraités nord-américains et européens qui séjournent dans ces îles lors de I'hiver boréal lorsqu'ils n'ont pas choisi de s'y établir définitivement.

Photo: Olivier Dehoorne

Pour la saison d'hiver 2006-2007, Vacances Signature proposait toujours la destination Costa Rica avec une offre d'hôtels qui représentait cependant une fraction de celle offerte dans l'axe Cuba-Mexique-République dominicaine. Malgré une croissance du marché des Canadiens sur l'ensemble de la zone caribéenne (+44\% de 2000 à 2004), leur présence demeure marginale (8\% en 2004) en comparaison des Étatsuniens $(52,4 \%)$ et des Européens qui représentent l'essentiel du marché émetteur pour les destinations de la Carailbe $^{2}$. En fait, le Canada représente à peu de choses près l'équivalent de ce que les Caribéens représentent eux-mêmes comme marché touristique pour la région. II importe donc de comprendre à l'échelle de quels pays la visite des Canadiens peu avoir un impact significatif.

Comme le soulignait récemment André Désiront (2006), pour les Québécois, l'espace touristique caribéen se résume au «triangle » des destinations que sont Cuba, la République dominicaine et l'axe Cancun / Riviera Maya au Mexique. Par ailleurs, en 2004, l'ensemble de la Caraïbe recevait la 


\section{Graphique 1}

\section{Évolution indicielle $(100=1980)$ des arrivées de touristes en provenance du Canada vers la Caraïbe (1980-2004)}

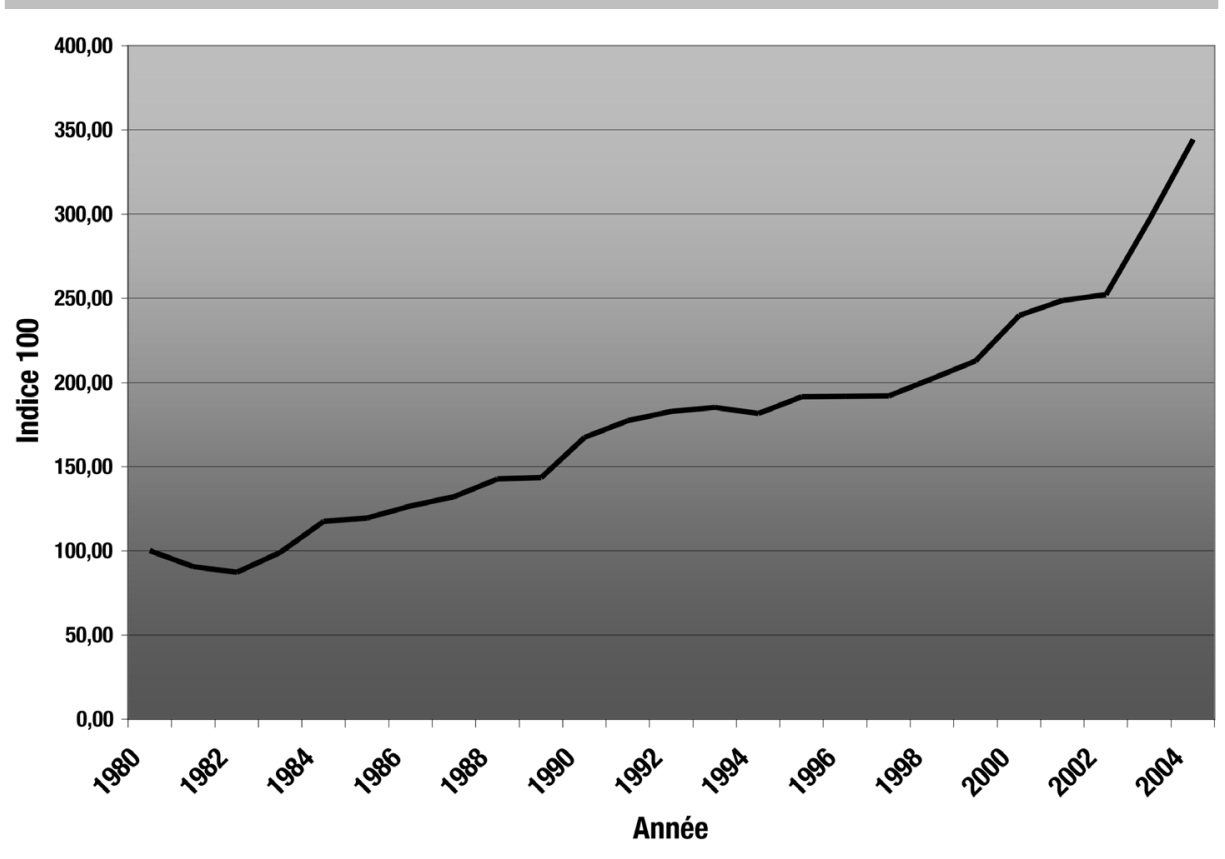

Source : Nos calculs à partir des données de la Caribbean Tourism Organization / CTO (2006) - tableau 2.

visite de 1,739 millions de touristes canadiens, dont 1,2 million concentrés seulement dans ce triangle (CTO, 2006 : tableaux 2, 14 et 15). Ces trois pays séparés de plusieurs milliers de kilomètres partagent cinq principales caractéristiques qui en font des pôles touristiques pratiquement indifférenciés: 1) la proximité (4 à 5 heures de vol des principales villes canadiennes) ; 2) la qualité de I'hébergement des grandes chaînes espagnoles et mexicaines ; 3) La qualité et la diversité des activités offertes sur place ; 4) la beauté des plages ; 5) la sécurité de la destination et des sites. Ces caractéristiques constituent l'image de marque de ces destinations auxquelles s'ajoutent des particularités locales associées à l'une ou l'autre des offres de services : accueil particulièrement chaleureux pour Cuba; accessibilité des sites culturels pour le Mexique; et prix imbattables pour la République dominicaine. Ces éléments constituent autant de catalyseurs d'arrivées touristiques - d'un certain type de visiteurs en fait - et nous proposons d'explorer brièvement en quoi consiste l'offre touristique de chacun de ces pays pour mieux comprendre pourquoi ils constituent le choix privilégié des Canadiens depuis plusieurs années.
Le tableau 1 présente le palmarès des cinq destinations les plus visitées en 2004 parmi lesquelles les trois pays du "triangle" se retrouvent. Malgré le quatrième rang qu'il occupe en volume d'arrivées, le cas particulier de Cuba mérite que l'on aborde cette destination d'entrée de jeu. Première industrie en importance dans l'île, le tourisme s'est développé de façon quasi exponentielle dans ce pays au cours de la décennie 1990. Récepteur de près de $10 \%$ de l'ensemble des arrivées à destination de la Carailbe en

\section{Tableau 1}

Arrivées de touristes dans les cinq destinations les plus visitées de la Caraïbe (2004)

\begin{tabular}{lcc} 
Pays & $\begin{array}{c}\text { Arrivées } \\
\text { (milliers) }\end{array}$ & $\begin{array}{c}\text { Parts de marché } \\
(\mathbf{\%})\end{array}$ \\
\hline Porto Rico & 3684,1 & 16,89 \\
\hline République dominicaine & 3443,2 & 15,78 \\
Mexique (Cancun) & 2331,4 & 10,69 \\
Cuba & 2048,6 & 9,39 \\
\hline Bahamas & 1561,3 & 7,16 \\
\hline
\end{tabular}

Source: Nos calculs à partir des données de la Caribbean Tourism Organization (2006) - tableau 5.
2004, Cuba affiche une « performance » enviable, considérant son inaccessibilité au principal marché émetteur que représentent les États-Unis avec plus de la moitié de toutes les arrivées dans la région en 2004. Si la nourriture laisse parfois à désirer, la qualité de l'offre d'hébergement des grandes chaînes espagnoles telles que Riu, lberostar, Melia, Bahia Principe, Bavaro et Occidental séduit le visiteur en quête d'une expérience... limitée! L'offre touristique de Varadero et des autres stations de l'île (Holguin, Cayo Coco, Cayo Largo, Cayo Santa Maria et Santa Lucia) mise sur la beauté des plages et sur la sécurité du site dont le régime castriste se porte garant, et pour cause... les dépenses touristiques représentaient en 2004 plus de 2 milliards USD (CTO, 2006 : 12). Sans ces revenus, le régime politique cubain aurait du mal à financer ses activités et les touristes le lui rendent bien... rares sont ceux qui intègrent la dimension politique à leur choix de destination, la sécurité étant considérée comme une valeur cardinale pour le tourisme, peu importe si le régime qui la procure constitue lui-même un "risque politique" (Sarrasin, 2004). Pour des raisons politiques encore une fois, la position dominante des Canadiens en termes de volume des arrivées et des investissements dans le secteur touristique cubain s'explique par l'embargo étatsunien qui dure depuis 1963 et qui empêche ceux-ci de se rendre directement dans l'île ou d'y investir. Cette situation pourrait changer à moyen terme, réduisant ainsi significativement l'influence des Européens et des Canadiens sur l'offre et la demande touristiques cubaines (Henthorne et Miller, 2003).

Le tableau 2 montre l'évolution des arrivées de touristes canadiens dans les destinations du «triangle " de la Caraïbe. On observe que la position de Cuba comme première destination des Canadiens s'est consolidée au cours de la période 2000-2004, en doublant pratiquement (+83\%) le nombre d'arrivées sur cinq ans. En l'absence des Étatsuniens, les Canadiens occupent donc avec les Espagnols une position dominante pour près de $28 \%$ des parts de marché. Le régime cubain a d'ailleurs intensifié sa présence publicitaire, particulièrement au Québec, au moment où les grossistes en voyages québécois multipliaient leur offre d'hôtels dans les enclaves touristiques cubaines au cours de la saison d'hiver 2006-2007. 
Tableau 2

Arrivées en provenance du Canada dans certains pays de la Caraïbe (en milliers, 2000-2004)

\begin{tabular}{lcccccc} 
& $\mathbf{2 0 0 0}$ & $\mathbf{2 0 0 1}$ & $\mathbf{2 0 0 2}$ & $\mathbf{2 0 0 3}$ & $\mathbf{2 0 0 4}$ & $\begin{array}{c}\text { Dépendance au } \\
\text { marché canadien } \\
\text { (2004) }\end{array}$ \\
\hline Cuba & 307,7 & 350,4 & 348,5 & 452,4 & 563,4 & $27,5 \%$ \\
République dominicaine & 245,7 & 283,5 & 313,5 & 412,6 & 448,6 & $13 \%$ \\
Cancun (Mexique) & 91,3 & 86,8 & 119,4 & 139,3 & 174,4 & $7,5 \%$ \\
\hline
\end{tabular}

Source : Caribbean Tourism Organization (2006) - tableaux 14 et 15.

La seconde destination touristique en importance du « triangle » est la République dominicaine. Affichant un volume d'arrivées de $20 \%$ moins élevé que Cuba en 2004, elle prend la position de tête en termes de croissance avec $+82 \%$ sur cinq ans. Partageant les caractéristiques des autres destinations du «triangle », elle propose cependant des forfaits "tout compris " en moyenne moins chers que ses deux principales concurrentes sur le marché canadien. Destination prisée des jeunes en quête d'une première expérience "Sud », la fragilité de son économie, sa dépendance au tourisme et le faible revenu moyen de ses habitants en font une destination souvent associée au tourisme sexuel, des hommes comme des femmes (Lévy et Lacombe, 2003). Partageant l'île d'Hispaniola avec Haïti, des épisodes de paludisme observés à la fin de l'année 2004 et au début de 2005 ont rappelé brutalement toute la fragilité du secteur (Public Health Agency of Canada, 2004). L'ouragan Wilma a placé la destination de Cancun au Mexique dans une situation similaire en novembre 2005, ce qui devrait nécessairement se traduire par une baisse marquée des arrivées, non seulement canadiennes, mais aussi internationales.

Troisième destination en importance du « triangle ", les Canadiens représentaient 7,5\% des arrivées à Cancun en 2004. Consacré pays de «l'Amérique du Nord » depuis la signature de l'Accord de libre-échange nordaméricain (ALENA) en 1994, les arrivées touristiques au Mexique témoignent de la forte dépendance du pays face aux ÉtatsUnis et laissent au Canada la place qui lui revient en termes statistiques, sa population représentant environ $10 \%$ de celle de son voisin du Sud. Affichant une croissance significative des arrivées durant la période 2000-2004 (+91\%), Cancun est la seule destination du «triangle » qui offre un nombre aussi élevé d'hôtels de 1000 chambres et plus. Dans ces conditions, l'offre touris- tique se décline sous la forme de véritables « petites villes » qui répondent à l'ensemble des besoins du visiteur, ce qui inclut non seulement l'hébergement et la nourriture, mais une pléthore de "services de divertissement » qui vont du shopping au cinéma, en passant par l'animation sur le site avec le résultat que la ville de Cancun... la vraie... s'est vidée graduellement de ses restaurants et de ses autres services connexes (hôtels, taxis, etc.), les «tout compris » contrôlant toujours davantage l'ensemble des besoins du touriste lors de son séjour, laissant aux petits acteurs locaux la partie congrue des retombées économiques du tourisme.

\section{Quelques impacts du tourisme de masse : la contribution du marché canadien}

Fortement concentré durant les mois de décembre à avril, les Canadiens sont en réalité majoritairement des Ontariens, comme en témoignent les données du tableau 3. Comptant pour près de $66 \%$ des arrivées canadiennes dans l'ensemble de la Caraïbe, le Québec, seconde province la plus peuplée du Canada, représentait tout juste $11 \%$ du marché en 2004.

\section{Tableau 3}

Touristes canadiens (par province) à destination de la Caraïbe (2003-2004)

\begin{tabular}{l|c|c|c|c}
\hline \multirow{2}{*}{} & \multicolumn{2}{|c|}{ Volume } & \multicolumn{2}{c}{ Pourcentage } \\
\cline { 2 - 5 } & $\mathbf{2 0 0 4}$ & $\mathbf{2 0 0 3}$ & $\mathbf{2 0 0 4}$ & $\mathbf{2 0 0 3}$ \\
\hline Alberta & 88,1 & 71,1 & 5,1 & 4,7 \\
Colombie-Britannique & 74,9 & 71,8 & 4,3 & 4,8 \\
Manitoba & 29,6 & 27,7 & 1,7 & 1,8 \\
Ontario & 1145,6 & 980,8 & 65,9 & 65,3 \\
Québec & 192,4 & 153,6 & 11,1 & 10,2 \\
Autres & 208,5 & 195,9 & 12 & 13,1 \\
Canada & 1739,1 & 1500,8 & 100 & 100 \\
\hline
\end{tabular}

Source : Caribbean Tourism Organization (2006) - tableau 13.
Les retombées de ces «visites » canadiennes ne sont pas différentes de celles des autres marchés en quête de " tout compris » à bas prix. Les pays de la Caraïbe se sont graduellement transformés en destinations touristiques, particulièrement depuis les années 1970 (République dominicaine et Cancun), et plus récemment (années 1990) dans le cas de Cuba. Le choix du tourisme, particulièrement enclavé dans la région par comparaison aux autres parties du monde comme l'Asie, a réduit le potentiel «multiplicateur » de l'activité touristique sur les autres secteurs de l'économie (Meyer, 2006 : 2). Les effets pervers du tourisme d'enclave ne sont pas nouveaux. Certains auteurs (Manning, 1982 ; Reid, 1992) suggèrent que les « tout compris » qui ne tentent pas minimalement de créer des liens économiques et culturels avec les communautés limitrophes consacrent la domination de la richesse étrangère sur la pauvreté autochtone. Dans le cas de la République dominicaine, particulièrement concernée par les écarts de revenus, certains auteurs ont associé le tourisme enclavé à des "camps de concentration du loisir " (Reid, 1992 : 75), en insistant sur le fait que le « modèle de développement » par enclave entretenait l'exploitation des classes sociales défavorisées par la création d'emplois peu rémunérés (Freitag, 1994). Dans ces conditions, la création d'emplois induite par le tourisme n'arrive pas à compenser ses effets négatifs, telle la croissance de l'inflation ou la spéculation foncière.

Le volume d'arrivées dans une destination et son éventuelle croissance, comme celle que nous avons observée pour le marché canadien au cours de la période 2000-2004, ne constituent donc pas une garantie de retombées économiques nettes. Pour Cuba, par exemple, seulement $30 \%$ à $38 \%$ des dépenses touristiques restent dans l'île (Duterme, 2006: 16). II ne s'agit donc pas seulement de faire que le visiteur dépense davantage pour son séjour en accroissant l'offre de services et la qualité des prestations, il importe que les dépenses touristiques aient un impact local accru sur l'emploi ou l'agriculture par exemple. Cette approche peut se réaliser difficilement dans le cadre du tourisme de masse «tout compris " tel que les Canadiens le pratiquent majoritairement dans les destinations du «triangle ". Les fuites (leakages) sont nombreuses et sont estimées par la Banque mondiale à 55 \% en moyenne des revenus bruts du tourisme dans les pays du Sud: 


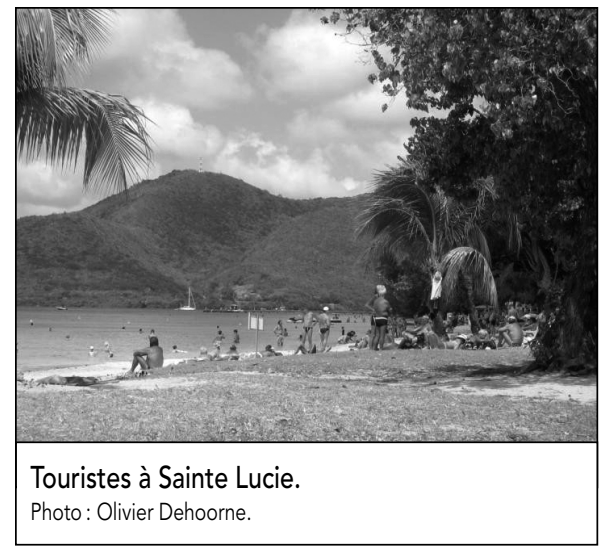

Leakages are particularly high in small developing country island economies, for many of which tourism is the principal export earner - examples are: St. Lucia 45\% [...], average for the Caribbean region is reported to be around 70\% [...] while a tourism official of the Bahamas states that in 1994 leakages for that country might have been as high as 90\% (Meyer, 2006 : 7).

L'analyse de Dorothea Meyer sur les effets du tourisme dans la Caraïbe montre que l'activité touristique induit un taux d'importation particulièrement élevé. Quatre principaux facteurs expliquent cette situation (idem : 8). D'abord, les petites économies insulaires dépendent davantage des importations, considérant leur capacité limitée de produire les biens et services dont l'industrie touristique a besoin. Ensuite, le développement de relations intersectorielles nationales solides exige des infrastructures importantes (routes, ports, aéroports) pour concurrencer efficacement les importations. Aussi, les pays de la Caraïbe ont-ils massivement choisi le tourisme comme secteur moteur de leur économie depuis au moins une décennie. Cela a consacré du même coup l'accélération des importations et la dépendance de ces pays face au marché mondial de produits et services. Enfin, la plupart des destinations de la zone caribéenne ont trop fortement misé exclusivement sur la croissance du tourisme en négligeant la croissance d'autres secteurs comme celui de l'agriculture ou de la transformation. Cela a notamment conduit à l'incapacité de l'agriculture locale à répondre efficacement (en volume et en coût de production) aux besoins du secteur touristique et à ceux de la population locale (Karagiannis, 2004).

\section{Conclusion}

L'objectif de cet article n'était pas de poser un regard complet et définitif sur le marché touristique que représentent les Canadiens dans les trois pays qualifiés de «triangle " touristique de la Caraïbe. L'analyse des séries statistiques, particulièrement pour la période 2000-2004, a cependant permis de dégager certaines lignes de force: en plus de choisir massivement quelques destinations de la région - cela s'applique particulièrement aux Québécois plutôt qu'aux autres Canadiens qui voyagent davantage dans les destinations anglophones -, l'affluence des touristes canadiens augmente en volume et en concentration... il y a donc toujours davantage de touristes canadiens qui choisissent toujours les mêmes destinations, pour le soleil, la mer et la plage. Ces pays du «triangle ", à l'instar des autres destinations de la région, offrent principalement des services intégrés verticalement, c'est-à-dire où les besoins du voyageur sont pris en charge du départ au retour, en passant par tous les aspects du séjour. Nous avons rappelé certaines limites des formules «tout compris", notamment pour l'économie des pays visités. Certaines destinations comme Cuba ou la République dominicaine offrent cependant d'autres expériences de séjour, par exemple les voyages à cheval ou les circuits organisés par Vélo Québec à Cuba, en solitaire ou en groupe. Ces «nouveaux produits" sont de plus en plus populaires auprès de la population québécoise. Les adeptes logent dans un petit hôtel local ; les itinéraires, réalisés à raison de quelques dizaines de kilomètres par jour, débutent souvent par Santiago de Cuba pour se terminer, deux semaines plus tard, à La Havane. Au lieu de l'expérience standardisée qu'offrent la plupart des « tout compris », un séjour à vélo offrira, pour le même prix qu'un forfait plage, des souvenirs de la Sierra de la Gran Piedra, de Guardalavaca, de Camaguey, des montagnes de Topes de Collantes, de Trinidad, de Santa Clara, de la péninsule Zapata, de Playa Larga dans la baie des Cochons et de la vallée de Vinales (Vincent, 2005). Bien que le tourisme de masse continuera vraisemblablement de croitre dans les destinations de la Caraïbe au cours des prochaines années, l'offre parallèle d'expériences touristiques «alternatives » pourra contribuer à l'accroissement des retombées positives du tourisme et nous croyons que les clientèles canadiennes peuvent jouer un rôle non négligeable dans ce processus.
Bruno Sarrasin est professeur au Département d'études urbaines et touristiques de l'Université du Québec à Montréal.

\section{Notes}

1 Moyenne géométrique.

2 Nos calculs à partir des données de la Caribbean Tourism Organization (2006 : tableau 9).

\section{Bibliographie}

"Le prix inhumain du sucre» (2006), FranceAntilles, jeudi 14 décembre, p. 6.

Bérubé, Gérard (2003), «Tourisme - Retour au beau fixe ", Le Devoir, 13 et 14 décembre.

Caribbean Tourism Organization (CTO) (2006), 2004 Full Annual Tourism Statistical Report, 20 novembre.

Désiront, André (2006), «Le triangle des Caraïbes ", La Presse, 12 octobre.

Duterme, Bernard (2006), «Expansion du tourisme international: Gagnants et perdants ", Alternatives Sud, vol. 13, n 3, p. 7-22.

Freitag, T.G. (1994), «Enclave Tourism Development: From Whom the Benefits Roll? ", Annals of Tourism Research, vol. 21, p. 538-554.

Henthorne, Tony L., et Mark M Miller (2003), "Cuban Tourism in the Caribbean Context: A Regional Impact Assessment", Journal of Travel Research, vol. 42, août, p. 84-93.

Karagiannis, N. (2004), « Dependence, Cumulative Causation and the Caribbean ", dans N. Karagiannis et M. Winter (dir.), The Caribbean Economies in an Era of Free Trade, Ashgate, Aldercroft.

Lévy, Joseph, et Élysabeth Lacombe (2003), " Le tourisme sexuel. Ses plaisirs et ses dangers ", Téoros, vol. 22, nº, printemps, p. 4-9.

Manning, F. (1982), «The Caribbean Experience ", Cultural Survival Quarterly, vol. 6, $\mathrm{n}^{\circ} 3$, p. 13-14.

Meyer, Dorothea (2006), Caribbean Tourism, Local Sourcing and Enterprise Development: Review of the Literature, Pro-poor Tourism Partnership Working Paper $n^{\circ} 18$, The Travel Foundation, janvier.

Public Health Agency of Canada (2004), "Reports of Malaria in the Dominican Republic ", Travel Health Advisory, 2 décembre.

Reid, A (1992). "Reflections. Waiting for Colombus ", The New Yorker, 24 février, p. 57-75. Cité par Freitag (1994) «Enclave Tourism Development: From Whom the Benefits Roll? ", Annals of Tourism Research, vol. 21, p. 538-554.

Sarrasin, Bruno (2004), « Risque politique et tourisme. Nouveautés et continuités ", Téoros, vol. 23, n 1, printemps, p. 12-22.

Vincent, Pierre (2005), "Cuba à vélo ", La Presse, 30 avril. 